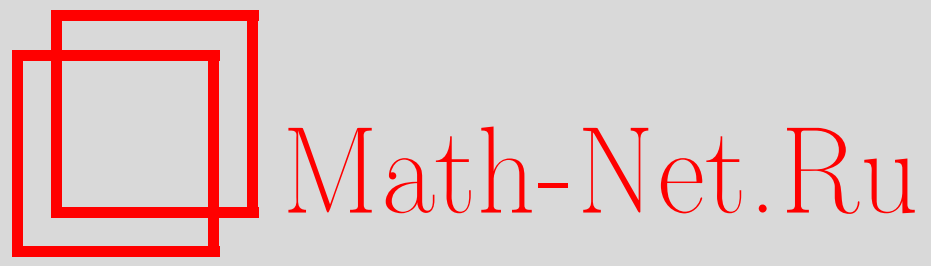

Ю. А. Куперин, С. Н. Набоко, Р. В. Романов, Спектральный анализ односкоростного оператора переноса и функциональная модель, Функи. анализ и его прил., 1999, том 33, выпуск 3, 47-58

DOI: https://doi.org/10.4213/faa365

Использование Общероссийского математического портала MathNet.Ru подразумевает, что вы прочитали и согласны с пользовательским соглашением

http://www . mathnet.ru/rus/agreement

Параметры загрузки:

IP : 52.6 .47 .48

26 апреля 2023 г., 11:40:11

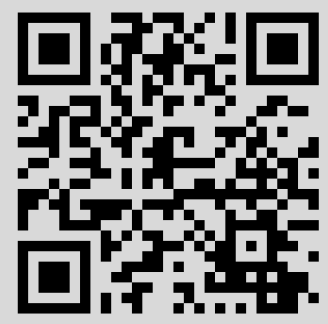


Функииональный анализ и его приложения

1999, т. 33, вып. 3, с. 47-58

УДК 517.948.35

\title{
Спектральный анализ односкоростного оператора переноса и функциональная модель*
}

\author{
(C) 1999. Ю. А. КупЕРин, С. Н. НАбоко, Р. В. Романов
}

\section{Введение}

Предлагаемая работа посвящена спектральному анализу несамосопряженного односкоростного оператора переноса, действующего в гильбертовом пространстве $L^{2}$ функций распределения. Известно [1], что во многих геометрических ситуациях этот оператор имеет богатый существенный спектр. Поэтому кажется разумным использовать для его спектрального анализа функциональную модель $[2,3,4]$. Мы будем изучать оператор, отвечающий переносу частиц в пластине из размножающего материала с изотропным распределением вторичных частиц, погруженной в однородный идеальный поглотитель. Фазовое пространство такой задачи - это множество $\Gamma=\mathbb{R} \times \Omega, \Omega \equiv[-1,1]$. Для частицы в точке $(x, \mu) \in \Gamma$ число $x \in \mathbb{R}$ - ее пространственная координата, а $\mu \in \Omega$ - косинус угла между скоростью частицы и координатной осью. Поглощение и размножение частиц характеризуются макроскопическим сечением $\sigma>0$, локальным коэффициентом размножения $c: \mathbb{R} \rightarrow \mathbb{R}_{+}$и оператором столкновений $K \in \mathbf{B} L^{2}(\Omega)$. Предполагается, что $c \in L^{\infty}(\mathbb{R})$.

Опишем результаты работы. В статье [5] было показано, что спектр оператора переноса в случае, когда функция $c$ пропорциональна индикатору интервала, состоит из конечного множества собственных значений, лежащих на мнимой оси, и непрерывного спектра, заполняющего вещественную ось. Мы выводим этот результат для произвольного финитного $c \in L^{\infty}(\mathbb{R})$ и даем аналог оценки Бирмана-Швингера для размерности подпространства, отвечающего дискретному спектру. Наш вывод в существенном следует работе [5], отличаясь некоторыми упрощениями и использованием современной терминологии. Далее, мы показываем, что непрерывный спектр оператора переноса является абсолютно непрерывным. Соответствующая компонента $T_{\text {ess }}$ этого оператора подобна самосопряженному оператору в случае, когда $c \notin \mathscr{E}$ для некоторого сингулярного множества $\mathscr{E} \subset L^{\infty}(\mathbb{R})$. Показано, что если $c \in \mathscr{E}$, то оператор переноса имеет единственную спектральную особенность в точке 0 . В этом случае компонента $T_{\text {ess }}$ подобна ортогональной сумме самосопряженного оператора и оператора со спектром конечной кратности $\mathscr{M}$, которая вычислена в терминах $c$. Для спектральной компоненты оператора переноса, отвечающей окрестности спектральной особенности, мы также даем оценку угла между соответствующими инвариантными подпространствами. Вывод этих результатов основан на выделении некоторых инвариантных подпространств модельного оператора, определенных при помощи спектрального разложения оператора $\Delta=1-S^{*} S$, где $S$

*Работа частично поддержана РФФИ, грант 97-01-01149, и ISSEP, грант а97-2400. 
- характеристическая функция. Оценка угла между этими подпространствами сводится к оценкам функции $S^{-1}$ на вещественной оси.

Следует подчеркнуть, что функциональная модель используется только в доказательствах и не участвует в формулировке результатов, относящихся к оператору переноса. Отметим также, что появление спектральной особенности в нашей задаче имеет совершенно непатологический характер. А именно, оказывается, что для любого финитного положительного $c \in L^{\infty}$ функция $\kappa c$ принадлежит $\mathscr{E}$ при некоторых значениях константы $\kappa$. Сказанное отличает оператор переноса от другого известного в математической физике примера оператора со спектральными особенностями - оператора Шрёдингера с комплексным потенциалом [6, 7], где спектральные особенности появляются ad hoc для специально выстроенных потенциалов.

Для удобства читателя в 1 дается краткое описание функциональной модели диссипативного оператора. Мы используем симметричную форму модели $[3,4]$. Далее мы излагаем конструкцию инвариантных подпространств, соответствующих абсолютно непрерывному спектру. Доказательства утверждений в абстрактной части работы (§1) за недостатком места опущены.

\section{§1. Функциональная модель}

Пусть $L$ - замкнутый диссипативный оператор с ограниченной мнимой частью $V=\operatorname{Im} L$, такой, что $\sigma_{\text {ess }}(L) \subset \mathbb{R}$, и пусть $E=\overline{\operatorname{Ran} V}$. Характеристической функцией $S$ оператора $L$ называется сжимающая аналитическая в $\mathbb{C}_{+}$ функция $S(z): E \rightarrow E$, определенная формулой

$$
S(z)=I+2 i \sqrt{V}\left(L^{*}-z\right)^{-1} \sqrt{V}, \quad z \in \mathbb{C}_{+} .
$$

По теореме Фату [2] $S$ обладает при п.в. $k \in \mathbb{R}$ граничными значениями $S(k) \equiv S(k+i 0)$. При $z \in \mathbb{C}_{+} \cap \rho(L)$ характеристическая функция обратима и $S^{-1}(z)=I-2 i \sqrt{V}(L-z)^{-1} \sqrt{V}$. Обозначим через $\mathscr{X}=L^{2}\left(\begin{array}{cc}I & S^{*} \\ S & I\end{array}\right)$ гильбертово пространство, полученное замыканием линеала $L^{2}(\mathbb{R}, E \oplus E)$ в метрике, заданной весом $\left(\begin{array}{cc}I & S^{*} \\ S & I\end{array}\right)$. Введем подпространство $\mathscr{K} \subset \mathscr{X}$ формулой

$$
\mathscr{K}=\left\{\left(\begin{array}{l}
\tilde{g} \\
g
\end{array}\right) \in \mathscr{X}: \tilde{g}+S^{*} g \in H_{-}^{2}(E), S \tilde{g}+g \in H_{+}^{2}(E)\right\} .
$$

Здесь $H_{ \pm}^{2}(E)$ - классы Харди аналитических в $\mathbb{C}_{ \pm} E$-значных функций $f$, таких, что $\sup _{\varepsilon>0} \int_{\mathbb{R}}\|f(k \pm i \varepsilon)\|_{E}^{2} d k<\infty$. Пусть $\mathscr{U}_{t}-$ унитарная группа сдвигов в $\mathscr{X},\left(\mathscr{U}_{t} f\right)(k)=e^{i k t} f(k)$. Тогда вполне несамосопряженная часть оператора $L$ унитарно эквивалентна генератору полугруппы сжатий $Z_{t}=\left.P_{\mathscr{K}} \mathscr{U}_{t}\right|_{\mathscr{K}}$, где $P_{\mathscr{K}}$ - ортопроектор на $\mathscr{K}$ в $\mathscr{X}$. Этот генератор называется функциональной моделью оператора $L$. Заметим, что, поскольку $S-$ сжимающая функция, $\Delta(k)=I-S^{*}(k) S(k) \geqslant 0$ при п. в. $k \in \mathbb{R}$. Это позволяет определить пространство $L^{2}(\mathbb{R} ; \Delta)$ как замыкание линеала $L^{2}(\mathbb{R}, E)$ в метрике, заданной весом $\Delta$. Далее $(\cdot, \cdot)$ обозначает угол между подпространствами гильбертова пространства. 
Мы используем без специальных пояснений утверждения о диссипативных операторах, соответствующие теоремам, сформулированным в книге [2] для сжатий.

Всюду в этой работе под инвариантным подпространством оператора понимается его регулярно инвариантное подпространство, т. е. подпространство $\mathscr{H}$ называется инвариантным подпространством оператора $L$, если $\overline{(L-z)^{-1} \mathscr{H}}=$ $\mathscr{H}$ при всех $z \in \rho(L)$. Используя функциональное исчисление для оператора $L$, можно показать, что каждое его регулярно инвариантное подпространство инвариантно в следуюшем естественном смысле: (i) $\operatorname{Dom}(L) \cap \mathscr{H}=(L-z)^{-1} \mathscr{H}$, $z \in \rho(L)$, и (ii) $L(\operatorname{Dom}(L) \cap \mathscr{H}) \subset \mathscr{H}$. Это позволяет определить замкнутое плотно заданное сужение $L_{\mathscr{H}}=\left.L\right|_{\mathscr{H}}$.

В дальнейшем некоторые инвариантные подпространства оператора определяются в терминах его функциональной модели. При этом оператор, осуществляющий унитарную эквивалентность вполне несамосопряженной части оператора $L$ и ее функциональной модели, в обозначениях опускается.

Определим абсолютно непрерывное подпространство $\mathscr{N}_{e} \subset \mathscr{K}$ оператора $L$ [3] как замыкание множества $\widetilde{\mathscr{N}}_{e}$ гладких векторов,

$$
\mathscr{N}_{e}=\overline{\widetilde{N}}_{e}, \quad \widetilde{\mathscr{N}}_{e} \equiv\left\{P_{\mathscr{K}}\left(\begin{array}{c}
\tilde{g} \\
-S \tilde{g}
\end{array}\right), \tilde{g} \in L^{2}(\mathbb{R} ; \Delta)\right\} .
$$

Определенное таким образом, $\mathscr{N}_{e}$ совпадает с инвариантным подпространством оператора $L$, отвечающим канонической факторизации $S=S_{i} S_{e}$ [2] характеристической функции. Будем называть спектр оператора $L$ абсолютно непрерывным, если $L=\left.\left.L\right|_{H_{0}} \oplus L\right|_{\mathscr{N}_{e}}$, где $H_{0}$ - инвариантное подпространство оператора $L$, такое, что $\left.L\right|_{H_{0}}$ - самосопряженный оператор с абсолютно непрерывным спектром в смысле спектральной теории [12].

Определим волновой оператор $W: L^{2}(\mathbb{R} ; \Delta) \rightarrow \mathscr{K}$ отношением $W: \tilde{g} \mapsto$ $P_{\mathscr{K}}\left(\begin{array}{c}\tilde{g} \\ -S \tilde{g}\end{array}\right)$. Как показано в [4], $W=\mathrm{s}-\lim _{t \rightarrow+\infty} e^{i L t} J e^{-i A_{0} t}$, где $J: L^{2}(\mathbb{R} ; \Delta) \rightarrow \mathscr{K}$ - некоторый изометричный оператор отождествления, для которого в модели имеется явная формула, а $A_{0}$ - оператор умножения на независимую переменную в $L^{2}(\mathbb{R} ; \Delta)$. Основное свойство гладких векторов выражается сплетающим соотношением

$$
(L-z)^{-1} W=W\left(A_{0}-z\right)^{-1}, \quad z \in \rho(L) .
$$

Следующая лемма дает описание некоторого класса инвариантных подпространств оператора $L$ в $\mathscr{N}_{e}$.

Пусть $\{X(k)\}$ - измеримое семейство подпространств пространства $E$, определенных при п. в. $k \in \mathbb{R}, X=\left\{f \in L^{2}(\mathbb{R}, E): f(k) \in X(k)\right.$ при п.в. $\left.k\right\}$. Положим $H=\bar{X}$ в $L^{2}(\mathbb{R} ; \Delta)$. Назовем кратностью спектра оператора $A$ число $m(A)=\inf \operatorname{dim} \mathscr{N}$, когда $\mathscr{N}$ пробегает порождающие подпространства оператора $A$.

Лемма 1. $\mathscr{H}=\overline{W H}$ - инвариантное подпространство оператора L. Если $S(k)$ обратима в широком смысле п. в. на вещественной оси, то

$$
m\left(L_{\mathscr{H}}\right)=\underset{k \in \mathbb{R}}{\operatorname{ess} \sup } \operatorname{dim} \Delta(k) X(k) .
$$

Eсли $\operatorname{ess} \sup _{k \in \mathbb{R}}\left\|\left(\left.S(k)\right|_{\Delta(k) X(k)}\right)^{-1}\right\|<\infty$, то $\left.W\right|_{H}$ ограниченно обратим и $L_{\mathscr{H}}$ подобен самосопряженному оператору. 
Отметим, что некоторый частный случай формулы (2) содержится в работе [8].

Задаваясь $X$, всегда можно построить второе инвариантное подпространство $\mathscr{H}^{\sim}$ оператора $L$ так, чтобы $\mathscr{N}_{e}=\overline{\mathscr{H} \dot{+} \mathscr{H}^{\sim}}$, применяя лемму $1 \mathrm{k}$ семейству $\left\{X(k)^{\perp}\right\}$. В общем случае, однако, неизвестна оценка угла $\left(\mathscr{H}, \mathscr{H}^{\sim}\right)$. Опишем способ выбрать $\{X(k)\}$ так, чтобы $\left(\mathscr{H}, \mathscr{H}^{\sim}\right)$ оценивался в терминах $S$.

Введем оператор $D(k)=S^{*}(k) S(k)$. Для данной измеримой функции $k \mapsto \gamma_{k}$, $\gamma_{k} \in[0,1]$, пусть $P_{1}(k), P_{2}(k)$ - спектральные проекторы оператора $D(k)$, отвечающие промежуткам $\left[0, \gamma_{k}\right),\left[\gamma_{k}, 1\right]$ соответственно и $X_{i}(k)=\operatorname{Ran} P_{i}(k)$, $i=1,2$, так что $E=X_{1}(k) \oplus X_{2}(k)$, поскольку $0 \leqslant D(k) \leqslant I$. Положим $X_{i}=$ $\left\{f \in L^{2}(\mathbb{R}, E): f(k) \in X_{i}(k)\right.$ при п. в. $\left.k \in \mathbb{R}\right\}, i=1,2$. По построению пара ортогональных подпространств $X_{1}, X_{2} \subset L^{2}(\mathbb{R}, E)$ приводит $\Delta$. Пусть $H_{i}=$ $\overline{X_{i}}, i=1,2$, в $L^{2}(\mathbb{R} ; \Delta)$, так что $L^{2}(\mathbb{R} ; \Delta)=H_{1} \oplus H_{2}$. Согласно лемме $1, \mathscr{H}_{i}=$ $\overline{W H_{i}}, i=1,2,-$ инвариантные подпространства оператора $L$.

Лемма 2. Имеет место неравенство

$$
\sin \left(\mathscr{H}_{1}, \mathscr{H}_{2}\right) \geqslant \underset{k \in \mathbb{R}}{\operatorname{essinf}}\left\|\left(\left.S(k)\right|_{X_{2}(k)}\right)^{-1}\right\|^{-1} .
$$

\section{$\S 2$. Линейный оператор переноса}

Далее $\chi_{M}(\cdot)$ - индикатор множества $M \subset \mathbb{R}, \mathbf{1}$ - индикатор множества $\Omega, f_{\infty}$ есть $L^{\infty}$-норма функции $f,\|\cdot\|_{2}$ - норма в классе Гильберта-Шмидта, $U_{\delta}(z)=\left\{z^{\prime} \in \mathbb{C}:\left|z-z^{\prime}\right|<\delta\right\}, \omega_{\delta}=U_{\delta}(0) \cap \mathbb{C}_{+}$и $d \mu-$ мера Лебега на $\Omega$. Введем класс $L_{0}^{+}=\left\{d \in L^{\infty}(\mathbb{R}): d(x) \geqslant 0\right.$ п. в. и существует $a$, такое, что $d(x)=0$ при п. в. $x$, для которых $|x|>a\}$.

Эволюция функции распределения в гильбертовом пространстве $H=L^{2}(\Gamma)$ описывается уравнением Больцмана [1]

$$
-i \partial_{t} u=L u \text {. }
$$

Ее генератор $L$, называемый односкоростным оператором переноса, имеет вид ${ }^{1}$

$$
L=i \mu \partial_{x}+i \sigma(1-c(x) K), \quad K=\frac{1}{2} \int_{\Omega} \cdot d \mu^{\prime} .
$$

Он действует в $H$ на области определения $\mathscr{D}=\{f \in H: f(\cdot, \mu)$ абсолютно непрерывна при п. в. $\mu \in \Omega$ и $\left.\mu \partial_{x} f \in H\right\}$ своей вещественной части $L_{0}=i \mu \partial_{x}$. Мнимая часть оператора $L$ ограничена. Оператор $L_{0}$ соответствует эволюции $U_{t}=\exp i L_{0} t,\left(U_{t} f\right)(x, \mu)=f(x-\mu t, \mu)$, функций распределения в вакууме.

Вместо $L$ удобно исследовать диссипативный оператор $T=L^{*}+i \sigma=i \mu \partial_{x}+$ $i \sigma c(x) K$. Не умаляя обшности, можно положить $\sigma=1$, так что $V=\operatorname{Im} T=$ $c(x) K$. Подпространство $\operatorname{Ran} K$ в $H$ естественно отождествляется с пространством $L^{2}(\mathbb{R})$ функций переменной $x: K H=L^{2}(\mathbb{R}) \otimes \mathbf{1} \simeq L^{2}(\mathbb{R})$.

\section{§3. Спектральный анализ оператора переноса}

Всюду ниже будем предполагать, что пластина имеет конечную ширину, т. е. $c \in L_{0}^{+}$. Далее, в зависимости от контекста, $\sqrt{c}$ обозначает либо оператор умножения на функцию $\sqrt{c(x)}$ в $K H=L^{2}(\mathbb{R})$, либо элемент из $E \equiv \overline{\operatorname{Ran} V} \subset K H$.

${ }^{1}$ Мы обозначаем через $K$ и сам оператор $K$ в $L^{2}(\Omega)$, и оператор $I \otimes K$ в $L^{2}(\mathbb{R} \times \Omega)=$ $L^{2}(\mathbb{R}) \otimes L^{2}(\Omega)$. 
Пусть $R(z)=(T-z)^{-1}$ и $R_{0}(z)=\left(L_{0}-z\right)^{-1}$. Умножая резольвентное тождество $R(z)-R_{0}(z)=-i R_{0}(z) V R(z)$ на $\sqrt{V}$, получим

$$
R(z)=R_{0}(z)-i R_{0}(z) \sqrt{V}\left(I+i \sqrt{V} R_{0}(z) \sqrt{V}\right)^{-1} \sqrt{V} R_{0}(z) .
$$

Отсюда видно, что $\sigma_{+}(T) \equiv \sigma(T) \cap \mathbb{C}_{+}=\{z:-1 \in \sigma(Q(z))\}$, где $Q(z)=$ $\left.i \sqrt{V} R_{0}(z) \sqrt{V}\right|_{\overline{\operatorname{Ran} V}}, z \in \mathbb{C}_{+}$, 一 оператор в пространстве $\overline{\operatorname{Ran} V} \subset L^{2}(\mathbb{R})$. Ядро оператора $Q(z)$ имеет вид (см. [5] $\left.]^{2}\right)-\frac{1}{2} \sqrt{c(x)} E(-i z|x-y|) \sqrt{c(y)}$, где $E(s)=$ $\int_{1}^{\infty} e^{-s t} d t / t$ при $\operatorname{Re} s>0$. Функция $E(s)$ допускает представление

$$
E(s)=-\ln s-\gamma+\theta(s),
$$

где $\theta(s)=-\sum_{m=1}^{\infty}(-s)^{m} /(m ! m)$ - целая функция, а $\gamma$ - постоянная Эйлера. Так каK $E(s)=O(\ln |s|)$ при $s \rightarrow 0$ и $c$ финитна, $Q(z)$ - оператор ГильбертаШмидта при любом $z \in \mathbb{C}_{+}$. Обозначим через $\left\{\eta_{n}(z)\right\}_{n=1}^{\infty},\left|\eta_{n}\right| \geqslant\left|\eta_{n+1}\right|>0$, собственные значения оператора $Q(z)$. Используя $(5)$, можно представить $Q(z)$ следующим образом:

$$
Q(z) \equiv \widetilde{Q}(z)+\frac{1}{2} \Theta(z)
$$

где $\widetilde{Q}(z)$ - оператор с ядром $\frac{1}{2} \sqrt{c(x)}(\ln (-i z|x-y|)+\gamma) \sqrt{c(y)}$, так, что $\Theta(z)$ — целая функция, а $\widetilde{Q}(z)$ - аналитическая функция в $\mathscr{O}=\mathbb{C} \backslash\{-i t, t \geqslant 0\}$. В соответствии с этой формулой $Q(z)$ допускает аналитическое продолжение из $\mathbb{C}_{+}$на $\mathscr{O}$.

Теорема 1. Пусть $c \in L_{0}^{+}$. Тогда невещественный спектр $\sigma_{+}(T)$ оператора $T$ - конечное множество собственных значений, лежаших на мнимой оси. Оператор $T$ не имеет присоединенньх векторов. Размерность $N(c)$ спектрального подпространства, отвечающего дискретному спектру, допускает оценку

$$
N(c) \leqslant 1+\frac{1}{4} \iint \ln ^{2}|x-y| c(x) c(y) d x d y .
$$

Существенный спектр $T$ совпадает с вещественной осъю: $\sigma_{\mathrm{ess}}(T)=\mathbb{R}$.

ДокаЗАтЕльство. Оператор $\sqrt{V} R_{0}(z)$ компактен в силу компактности оператора $Q(z)$, поскольку

$$
\operatorname{Re} Q(z)=\frac{i}{2}\left(\sqrt{V}\left(R_{0}(z)-R_{0}(\bar{z})\right) \sqrt{V}\right)=-\operatorname{Im} z \sqrt{V} R_{0}(z) \cdot R_{0}(\bar{z}) \sqrt{V}
$$

Отсюда ввиду резольвентного тождества вытекает компактность разности $R(z)-R_{0}(z)$ при $z \in \rho(T), \operatorname{Im} z \neq 0$. Стало быть, $\sigma_{+}(T)$ дискретно в $\mathbb{C}_{+}$и $\sigma_{\text {ess }}(T)=\mathbb{R}$ в силу теоремы Вейля [12].

Далее, $\eta_{n}(z) \notin \mathbb{R}$ при всех $n$ (и, в частности, $\left.-1 \notin \sigma(Q(z))\right)$, если $k \equiv \operatorname{Re} z \neq 0$. Достаточно показать, что оператор $\operatorname{Im} Q(z)$ строго положителен или отрицателен при $k \neq 0$. Рассмотрим оператор $\Xi(z)=\left.i K R_{0}(z)\right|_{K H}$, действующий в

2 Это интегральное ядро вычислено в $[5, \S 2]$ для случая $c=c_{\infty} \chi_{[-a, a]}$. Для произвольного $c \in L_{0}^{+}$результат получается очевидным преобразованием. Далее мы используем некоторые вспомогательные оценки для $Q$, полученные в $[9,10]$ для случая $c=c_{\infty} \chi_{[-a, a]}$ при нахождении спектра, поскольку в общем случае их доказательства в точности те же самые. 
пространстве $K H$. В представлении Фурье по переменной $x$ оператор $\operatorname{Im} \Xi(z)$ действует как умножение на функцию

$$
r_{z}(p)=\frac{1}{4 p} \ln \frac{(p-k)^{2}+(\operatorname{Im} z)^{2}}{(p+k)^{2}+(\operatorname{Im} z)^{2}} .
$$

Ясно, что $r_{z}(p)<0$ или $-r_{z}(p)<0$ и, таким образом, $\operatorname{Im} \Xi(z)<0$ или $-\operatorname{Im} \Xi(z)<0$ в зависимости от $\operatorname{sign} k$. Так как $\operatorname{Im} Q(z)=\left.\sqrt{c} \operatorname{Im} \Xi(z) \sqrt{c}\right|_{E}$, это дает $\operatorname{Im} Q(z)<0$ или $-\operatorname{Im} Q(z)<0$. Поэтому $\sigma_{+}(T) \subset i \mathbb{R}$.

Пусть $z=i \varepsilon, \varepsilon>0$. В представлении Фурье $\Xi(i \varepsilon)=\Xi^{*}(i \varepsilon)$ действует как умножение на функцию

$$
\xi_{\varepsilon}(p)=-\frac{\varepsilon}{2} \int_{-1}^{1} \frac{d \mu}{|p \mu+i \varepsilon|^{2}}=-\frac{1}{|p|} \arctan \frac{|p|}{\varepsilon} .
$$

Таким образом, $Q(i \varepsilon)=\left.\sqrt{c} \Xi(i \varepsilon) \sqrt{c}\right|_{E}-$ отрицательный оператор, монотонно возрастающий вместе с $\varepsilon$. Рассмотрим $Q^{\prime}(i \varepsilon)=-\left.i \sqrt{c}(d \Xi(i \varepsilon) / d \varepsilon) \sqrt{c}\right|_{E}$. В представлении Фурье оператор в круглых скобках действует как умножение на функцию $\left(p^{2}+\varepsilon^{2}\right)^{-1}$. Таким образом, $i Q^{\prime}(i \varepsilon)>0$. Стандартные аргументы теории возмущений позволяют отсюда заключить, что $(I+Q(z))^{-1}$ имеет простые полюсы в точках спектра $\sigma_{+}(T)$, и то же верно для $R(z)$ по (4). Стало быть, $T$ не имеет присоединенных векторов.

Оценим $N(c)$. Обозначим через $N_{\varepsilon}(c)$ ранг спектрального проектора, отвечающего промежутку $[i \varepsilon, i \infty)$. Поскольку $\sigma_{+}(T)$ дискретно в $\mathbb{C}_{+}, N_{\varepsilon}(c)<$ $\infty$ при $\varepsilon>0$. В силу монотонности и непрерывности функций $\eta_{n}(i \varepsilon)$ имеем $N_{\varepsilon}(c) \equiv \#\{s \geqslant \varepsilon: \operatorname{ker}(I+Q(i s)) \neq 0\}=\#\{\tau \in(0,1]: \operatorname{ker}(I+\tau Q(i \varepsilon)) \neq 0\} \mathrm{c}$ учетом кратности. Далее, согласно (6),

$$
2 Q(i \varepsilon)=Q^{0}+\Theta(i \varepsilon)+(\gamma+\ln \varepsilon) \mathbf{P}_{1} \equiv 2 Q_{1}(i \varepsilon)+(\gamma+\ln \varepsilon) \mathbf{P}_{1},
$$

где $\mathbf{P}_{1}=\langle\cdot, \sqrt{c}\rangle \sqrt{c},\langle\cdot, \cdot\rangle$ - скалярное произведение в $L^{2}(\mathbb{R})$, а $Q^{0}$ - оператор с ядром $\sqrt{c(x) c(y)} \ln |x-y|$. Поскольку $\operatorname{rank} \mathbf{P}_{1}=1$, это дает $\#\{\tau \in(0,1]$ : $\operatorname{ker}(I+\tau Q(i \varepsilon)) \neq 0\} \leqslant 1+\#\left\{\tau \in(0,1]: \operatorname{ker}\left(I+\tau Q_{1}(i \varepsilon)\right) \neq 0\right\} \leqslant 1+\left\|Q_{1}(i \varepsilon)\right\|_{2}^{2}$. Воспользуемся теперь следующей оценкой для $\Theta(z)$ (см. (5), (6)):

$$
\|\Theta(z)\|_{2} \leqslant \sum_{1}^{\infty} \frac{\left\|A_{m}\right\|_{2}|z|^{m}}{m ! m} \leqslant c_{1} \sum_{1}^{\infty} \frac{(2 a|z|)^{m}}{m ! m} \leqslant c_{1} \sum_{1}^{\infty} \frac{(2 a|z|)^{m}}{m !}=c_{1}\left(e^{2 a|z|}-1\right),
$$

где $c_{1}=\int_{\mathbb{R}} c(x) d x$ и $A_{m}$ - оператор с ядром $\sqrt{c(x)}|x-y|^{m} \sqrt{c(y)}$, для которого справедлива оценка $\left\|A_{m}\right\|_{2}^{2}=\iint|x-y|^{2 m} c(x) c(y) d x d y \leqslant(2 a)^{2 m} c_{1}^{2}$. Это дает

$$
N_{\varepsilon}(c) \leqslant 1+\left\|Q_{1}(i \varepsilon)\right\|_{2}^{2}=1+\frac{1}{4}\left\|Q^{0}\right\|_{2}^{2}+O(\varepsilon) .
$$

Так как $N(c)=\lim _{\varepsilon \downarrow 0} N_{\varepsilon}(c)$, мы приходим к $(7)$.

Отметим, что $\sigma_{+}(T)$ непусто при любой ненулевой функции $c \in L_{0}^{+}$, так как $\|Q(i t)\| \rightarrow \infty$ при $t \rightarrow 0$ и $\|Q(i t)\| \rightarrow 0$ при $t \rightarrow \infty$.

Доказательство конечности $N(c)$, данное в работах $[5,9]$ для случая $c=$ $c_{\infty} \chi_{[-a, a]}$, основано на тех же обстоятельствах (монотонность функции $Q(i \varepsilon)$ и конечность ранга расходящегося слагаемого в асимптотике функции $Q(i \varepsilon)$ в 0), что и приведенное выше. Оценка размерности (7) является, по-видимому, новой. 
Разложим пространство $H$ в линейную сумму инвариантных подпространств, соответствующих компонентам спектра $\sigma(T): H=H_{d} \dot{+} H_{\mathrm{ess}}$, где $H_{d}=\mathscr{P}_{d} H, H_{\mathrm{ess}}=\left(I-\mathscr{P}_{d}\right) H, \mathscr{P}_{d}$ - риссовский проектор, отвечающий $\sigma_{+}(T)$. По теореме 1 пространство $H_{d}$ конечномерно и, стало быть, $\left(H_{d}, H_{\mathrm{ess}}\right)>0$. Приведем без доказательства оценку угла $\left(H_{d}, H_{\mathrm{ess}}\right)$ для малых $c$.

ПРЕДЛОЖЕНИЕ 1. Пусть с такова, что интеграл в (7) меньше 4 и, стало бълть, $\operatorname{dim} H_{d}=1$. Тогда

$$
\sin \left(H_{d}, H_{\mathrm{ess}}\right) \geqslant \frac{e^{-\gamma}}{4+\pi^{2}} \frac{1}{a c_{\infty}} \exp \left(-\frac{2}{c_{1}}\right) .
$$

Перейдем к изучению компоненты $T_{\mathrm{ess}}=\left.T\right|_{H_{\mathrm{ess}}}$ средствами функциональной модели.

Заметим, что $\operatorname{Re} Q(z) \leqslant 0$ при $z \in \mathbb{C}_{+}$по определению оператора $Q$ и, стало быть, $I-Q(z)$ имеет ограниченный обратный, $\left\|(I-Q(z))^{-1}\right\| \leqslant 1$. При $z \in \mathbb{C}_{+}$ характеристическая функция $S(z)$ оператора $T$ выражается через $Q$ следующим образом [11]:

$$
S(z)=\frac{I+Q(z)}{I-Q(z)} .
$$

Отсюда видно, что $S$ допускает аналитическое продолжение на открытое множество, содержащее $\mathbb{R} \backslash 0$. Следующая лемма сводит, до некоторой степени, изучение оператор-функции $S$ к изучению скалярной аналитической функции. Напомним, что скалярная аналитическая функция $f$ называется скалярным кратным [2] для $S$, если существует ограниченная аналитическая в $\mathbb{C}_{+}$функция $\Pi$, такая, что $f(z) I=S(z) \Pi(z)=\Pi(z) S(z)$ при $z \in \mathbb{C}_{+}$.

ЛЕмма 3. S обладает скалярньлм кратнылм.

ДокАЗАтЕльство. Из $(11)$ видно, что $T(z) \equiv S(z)-I$ принадлежит классу $\mathbf{S}^{2}$ вместе с $Q(z)$ при всех $z \in \mathbb{C}_{+}$. Покажем, что $\|T(z)\|_{2}$ равномерно ограничена в $\mathbb{C}_{+}$. В самом деле, согласно $(6), T(z)=(A+\Theta(z))(1-Q(z))^{-1}+M(z)$, где $\operatorname{rank} M(z)=1$ и $\|A\|_{2}<\infty$. Поскольку $\|T(z)\| \leqslant 1+\|S(z)\| \leqslant 2$, мы имеем $\|M(z)\|_{2}=\|M(z)\| \leqslant 2+\|A\|+C_{\delta}|z| \leqslant C_{1, \delta}$ при $|z| \leqslant \delta$, что дает $\sup _{z \in \omega_{\delta}}\|T(z)\|_{2} \leqslant \widetilde{C}_{\delta}<\infty$, так как $\|\Theta(z)\|_{2} \leqslant C_{\delta}|z|$ при $|z| \leqslant \delta$ по (10). Лемма 5 работы [10] утверждает, в наших обозначениях, что ${ }^{3}$

$$
\|Q(z)\|_{2}^{2} \leqslant \frac{C_{\varepsilon}}{1+|\operatorname{Re} z|}
$$

при $z \in \Pi_{\varepsilon} \cap\{z:|\operatorname{Re} z| \geqslant 1\}$ для любого $\varepsilon>0$, где $\Pi_{\varepsilon}=\{z: 0 \leqslant \operatorname{Im} z \leqslant \varepsilon\}$. Зафиксировав произвольное $\varepsilon \in(0,1]$ и выбрав $\delta>\sqrt{2}$, получим $\|T(z)\|_{2} \leqslant$ $C<\infty$ при $z \in \Pi_{\varepsilon}$. Далее, ввиду очевидного неравенства $|E(s)| \leqslant E(\operatorname{Re} s) \leqslant$ $E\left(\operatorname{Re} s_{0}\right)$, справедливого при $0<\operatorname{Re} s_{0} \leqslant \operatorname{Re} s,\|Q(z)\|_{2} \leqslant\|Q(i \varepsilon)\|_{2}<\infty$ при $z \in \mathbb{C}_{+} \backslash \Pi_{\varepsilon}$. Собирая вместе полученные оценки, имеем $\sup _{z \in \mathbb{C}_{+}}\|T(z)\|_{2}<$ $\infty$. Пусть $m(z) \equiv \operatorname{det}\left(I-T^{2}(z)\right)$. Воспользуемся следующим утверждением, по сушеству доказанным в [13]. Если $A(\cdot)$ есть $\mathbf{S}^{2}$-значная функция на области $\mathscr{D} \subset \mathbb{C}$, аналитическая в операторной норме, и $\sup _{z \in \mathscr{D}}\|A(z)\|_{2}<\infty$, то $s(z)=$ $\operatorname{det}\left(I-A^{2}(z)\right)$ является скалярным кратным для $S(z)=I+A(z)$, если $s(z) \not \equiv 0$.

${ }^{3}$ Эта оценка была фактически получена в ходе доказательства. Формулировка утверждения в работе [10] ослаблена до оценки операторной нормы оператора $Q(z)$. 
Поскольку $T(\cdot)$, очевидно, аналитична, отсюда заключаем, что $m$ является скалярным кратным для $S$.

Оценка (12) использовалась в [10] для вывода некоторых поточечных асимптотик решений уравнения Больцмана. Следует отметить, что сам этот вывод содержит ошибку: логарифмическая оценка для $(I+Q(z))^{-1}$ в окрестности точки 0, даваемая леммой 6 этой работы, вообше говоря, не выполняется.

Пусть $\mathscr{B}_{c}=\left\{k_{n}=\lim _{\varepsilon \downarrow 0} \eta_{n}(i \varepsilon):\left|k_{n}\right|<\infty\right\}$. Определим сингулярное множество $\mathscr{E}$ следующим образом: $\mathscr{E}=\left\{c \in L_{0}^{+}:-1 \in \mathscr{B}_{c}\right\}$. Отметим, что при любом $c \in L_{0}^{+}$функция $\kappa c$ принадлежит $\mathscr{E}$ для бесконечного набора $\left\{-k_{n}^{-1}, k_{n} \in \mathscr{B}_{c}\right\}$ значений константы $\kappa>0$. Следующая лемма мотивирует выделение множества $\mathscr{E}$.

Лемма 4. 1. Если $c \in \mathscr{E}$, то существует функиия $f:(0,1) \rightarrow\{u \in E$, $\|u\|=1\}$, такал, что $S(i \rho) f(\rho) \rightarrow 0$ при $\rho \rightarrow 0$.

2. Если с $\notin \mathscr{E}$, то $\sup _{z \in \omega_{\delta}}\left\|S^{-1}(z)\right\|<\infty$ при достаточно малом $\delta>0$.

ДокАЗАТЕЛЬСтво. 1. По определению множества $\mathscr{E}$ существует нормированная собственная функция $\varphi_{\rho}$ оператора $Q(i \rho), Q(i \rho) \varphi_{\rho}=\eta(\rho) \varphi_{\rho}$, такая, что $\eta(\rho) \rightarrow-1$ при $\rho \rightarrow 0$. Достаточно положить $f(\rho)=\varphi_{\rho}$.

2. Согласно (11), имеем $S^{-1}(z)=-I+2(I+Q(z))^{-1}$ при $z \in \mathbb{C}_{+} \backslash \sigma_{+}$и, таким образом, требуемая оценка эквивалентна конечности $\sup _{z \in \omega_{\delta}}\left\|(I+Q(z))^{-1}\right\|$. Предположим, что $\left(I+Q\left(z_{n}\right)\right) \varphi_{n} \rightarrow 0$ при $z_{n} \rightarrow 0$ для некоторой последовательности $\left\{\varphi_{n}\right\},\left\|\varphi_{n}\right\|=1$. Тогда $\left\langle\operatorname{Im} Q\left(z_{n}\right) \varphi_{n}, \varphi_{n}\right\rangle \rightarrow 0$. Tак как $\operatorname{Im} Q(z)$ является знакоопределенной при $\operatorname{Re} z \neq 0$ и $\operatorname{Im} Q(z)=0$ при $\operatorname{Re} z=0$, то $\operatorname{Im} Q\left(z_{n}\right) \varphi_{n} \rightarrow 0$ и, следовательно, $\left(I+\operatorname{Re} Q\left(z_{n}\right)\right) \varphi_{n} \rightarrow 0$. Согласно $(6), \operatorname{Re} Q(z)-$ $Q(i|z|) \rightarrow 0$ в операторной норме при $z \rightarrow 0$. Таким образом, $\left(I+Q\left(i\left|z_{n}\right|\right)\right) \varphi_{n}$ $\rightarrow 0$, что противоречит условию $c \notin \mathscr{E}$.

Таким образом, при $c \in \mathscr{E}$ функция $S^{-1}(\cdot)$ не ограничена в окрестности 0 . Имеется две a priori допустимых причины такого поведения: наличие «сингулярного» внутреннего сомножителя в канонической факторизации функции $S$ и/или спектральная особенность в точке 0. Следуя [3], мы называем точку $k \in \mathbb{R}$ спектральной особенностью, если $\sup _{z \in U_{\delta}}(k) \cap \mathbb{C}_{+}\left\|S_{e}^{-1}(z)\right\|=\infty$ при всех $\delta>0$. Ниже, в предложении 2, мы покажем, что реализуется только вторая возможность. Предварительно получим некоторое явное описание множества $\mathscr{E}$.

ЛЕмма 5. $S(\cdot)$ непрерывна в 0 в операторной норме, $и$

$$
c \in \mathscr{E} \Longleftrightarrow \operatorname{ker}\left(I-\widetilde{Q}_{0}^{2}-\frac{2}{\vartheta_{c}}\langle\cdot, \sqrt{c}\rangle \sqrt{c}\right) \neq 0,
$$

где $\widetilde{Q}_{0}$ - интегральньй оператор слдром $\frac{1}{2} \sqrt{c(x)} \ln (|x-y| /(2 a)) \sqrt{c(y)}, a \vartheta_{c}=$ $\left\langle\left(I-\widetilde{Q}_{0}\right)^{-1} \sqrt{c}, \sqrt{c}\right\rangle$.

ДокАЗАтЕльство. Поскольку $\|\Theta(z)\|=o(1)$ при $z \rightarrow 0$, из (6) следует, что $(I-\widetilde{Q}(z))^{-1}$ существует и ограничен, если $z \in \omega_{\delta}$ при достаточно малом $\delta$. Из тождества

$$
(I-Q(z))^{-1}-(I-\widetilde{Q}(z))^{-1}=-(I-Q(z))^{-1} \frac{\Theta(z)}{2}(I-\widetilde{Q}(z))^{-1}
$$

видно, что $S$ непрерывна в 0 , если это верно для $(I-\widetilde{Q}(z))^{-1}$, так как $S(z)=$ $-I+2(I-Q(z))^{-1}$ по (11). Воспользуемся теперь леммой 7 работы [9], которая 
утверждает, в наших обозначениях, что $\widetilde{Q}_{0}<0$, и, стало быть, оператор $I-\widetilde{Q}_{0}$ обратим, и $\vartheta_{c} \neq 0$. Пусть $\alpha(z)=\frac{1}{2} \ln \left(-2 a e^{\gamma} i z\right)$. Исходя из формулы $\widetilde{Q}(z)=$ $\widetilde{Q}_{0}+\alpha(z) \mathbf{P}_{1}$, прямым вычислением получаем

$$
(I-\widetilde{Q}(z))^{-1}=\left(I-\widetilde{Q}_{0}\right)^{-1}+\frac{\alpha(z)}{1-\alpha(z) \vartheta_{c}}\left(I-\widetilde{Q}_{0}\right)^{-1} \mathbf{P}_{1}\left(I-\widetilde{Q}_{0}\right)^{-1} .
$$

Таким образом, $S$ непрерывна в 0 и

$$
S(0)=\frac{I+\widetilde{Q}_{0}}{I-\widetilde{Q}_{0}}-\frac{2}{\vartheta_{c}}\left(I-\widetilde{Q}_{0}\right)^{-1} \mathbf{P}_{1}\left(I-\widetilde{Q}_{0}\right)^{-1} .
$$

Умножая это равенство справа и слева на $I-\widetilde{Q}_{0}$, получим $(13)$, так как $c \in \mathscr{E}$, если и только если $\operatorname{ker} S(0) \neq 0$.

ПрЕДлОЖЕНИЕ 2. Компонента $T_{\mathrm{ess}}$ оператора $T$ имеет только абсолютно непрерьвньй спектр.

ДокАЗАТЕльство. Заметим, что скалярное кратное $m$ допускает аналитическое продолжение на открытую окрестность $\mathbb{R} \backslash 0$, поскольку такое продолжение допускает $Q(z)$ как $\mathbf{S}^{2}$-значная оператор-функция. Это означает, что сингулярный сомножитель $m_{s}$ в канонической факторизации функции $m$ имеет вид $m_{s}(z)=e^{i \mu_{1} z-\mu_{2} i / z}$ с некоторыми $\mu_{1}, \mu_{2} \geqslant 0$.

Из формулы $(15)$ видно, что $(I-\widetilde{Q}(z))^{-1}$ является ограниченной аналитической функцией в $U_{\tau}(0) \cap \mathscr{O}, \tau=\left(2 a e^{\gamma}\right)^{-1}$. Разрешая тождество (14), справедливое при $z \in \omega_{\tau}$, относительно $(I-Q(z))^{-1}$ и учитывая, что $\|\Theta(z)\|=o(1)$ при $z \rightarrow 0$, получим, что $(I-Q(z))^{-1}$, а с ней и $S(z)$, допускает ограниченное аналитическое продолжение на $U_{\delta}(0) \cap \mathscr{O}$ при достаточно малом $\delta$. Воспроизводя рассуждения из начала доказательства леммы 3 , получим отсюда, что $m$ допускает аналитическое продолжение на $U_{\delta}(0) \cap \mathscr{O}$, которое ограничено ввиду неравенства $\left|\operatorname{det}\left(I-A^{2}\right)\right| \leqslant \exp \left(\|A\|_{2}^{2}\right), A \in \mathbf{S}^{2}$. Отсюда следует, что $\mu_{2}=0$. В самом деле, в противном случае, применяя теорему Карлсона [12] к функции $g(1 / z)$, где $g(z)=e^{\mu_{2} i /(2 z)} m(z)$, получим $g \equiv 0$.

Далее, $\|Q(i \varepsilon)\|_{2} \rightarrow 0$ при $\varepsilon \rightarrow \infty$ по теореме Лебега. Отсюда следует, что $m(i \varepsilon) \rightarrow 1$ при $\varepsilon \rightarrow \infty$. Это дает $\mu_{1}=0$. Наконец, $m(z)=0$ при $z \in \mathbb{C}_{+}$, если и только если, $z \in \sigma_{+}$. В самом деле, $m(z)=\operatorname{det}(S(z)(2+S(z)))$, поэтому $m(z)=0$, если либо $\operatorname{ker} S(z) \neq 0$, либо $\operatorname{ker}(2+S(z)) \neq 0$. Второе невозможно, поскольку $\|S(z)\| \leqslant 1$. Таким образом, каноническая факторизация функции $m$ имеет вид $m=b m_{e}$, где $m_{e}$ - внешняя функция, а $b$ - конечное произведение Бляшке, отвечающее множеству $\sigma_{+}$. Пусть $S=B \widetilde{S}-$ каноническая факторизация функции $S$. Сомножитель $\widetilde{S}$ совпадает с характеристической функцией оператора $\widetilde{T}_{\mathrm{ess}}=\left.T\right|_{\mathscr{N}_{e}}$, где $\mathscr{N}_{e}-$ абсолютно непрерывное подпространство оператора $T$ [3]. Поскольку $m_{e}$ - скалярное кратное для $\widetilde{S}[2$, Предложение V.6.4], имеем $\sigma\left(\widetilde{T}_{\mathrm{ess}}\right) \subset \mathbb{R}$, и, таким образом, $\widetilde{T}_{\mathrm{ess}} \subset T_{\mathrm{ess}} \cdot$

Остается заметить, что $\mathscr{N}_{e}=H_{\mathrm{ess}} \ominus H_{0}$. В самом деле, поскольку $\left(H_{d}, H_{\mathrm{ess}}\right)>$ 0 , имеем $H \ominus H_{0}=\mathscr{N}_{e}+\mathscr{N}_{i}$, где $\mathscr{N}_{i}$ - внутреннее подпространство оператора $T$ (см. [3]). В нашем случае $\mathscr{N}_{i}=H_{d}$, откуда и следует требуемое равенство.

Таким образом, точка 0 при $c \in \mathscr{E}$ является спектральной особенностью оператора $T$. В соответствии с критерием Надя-Фояша [2] это означает, что при $c \in \mathscr{E}$ оператор $T_{\text {ess }}$ не подобен самосопряженному. 
ПРЕДЛОЖеНИЕ 3. Пусть $с \notin \mathscr{E}$. Тогда $T_{\mathrm{ess}}$ подобен самосопряженному опеpamopy.

ДокаЗАтЕльство. Заметим, во-первых, что $\sup _{z \in X}\left\|\widetilde{S}^{-1}(z)\right\|<\infty$ для любого компакта $X \subset \mathbb{C}_{+}$, поскольку $\sigma\left(T_{\mathrm{ess}}\right) \subset \mathbb{R}$. Далее, $\|Q(z)\| \leqslant\|Q(i \operatorname{Im} z)\| \rightarrow 0$ при $\operatorname{Im} z \rightarrow \infty$. Отсюда и из оценки (12) заключаем, что $S(z) \rightarrow I$ в операторной норме при $z \rightarrow \infty$ в $\mathbb{C}_{+}$равномерно по $\arg z$. Переходя в (8) к пределу при $\operatorname{Im} z \rightarrow 0$, убеждаемся, что, в зависимости от $\operatorname{sign} k$, либо $\operatorname{Im} Q(k)<0$ либо $-\operatorname{Im} Q(k)<0$ и, стало быть, $\operatorname{ker} S(k)=0$ при $k \neq 0$. Собирая вместе полученные утверждения, заключаем, что $\sup _{z \in \mathbb{C}_{+} \mid \omega_{\delta}}\left\|\widetilde{S}^{-1}(z)\right\|<\infty$, так как $\widetilde{S}^{-1}=S^{-1} B$, где $B-$ сжатие. Отметим, что этот вывод не зависит от предположения $c \notin \mathscr{E}$. Наконец, учитывая п. 2 леммы 4 , получаем, что $\sup _{z \in \mathbb{C}_{+}}\left\|\widetilde{S}^{-1}(z)\right\|<\infty$, и доказываемый результат следует из критерия Надя-Фояша.

На абстрактном уровне, всегда можно разложить $T_{\mathrm{ess}}$ в линейную сумму $T_{\text {ess }}=T_{1} \dot{+} T_{2}$, компонента $T_{2}$ которой подобна самосопряженному оператору, полагая $\gamma_{k} \equiv \beta, \beta \in(0,1)$, в конструкции $\S 1$. Если $S(z) \in I+\mathbb{S}^{\infty}$ и функция $S$ непрерывна вплоть до вещественной оси и на бесконечности по операторной норме, как это и происходит в рассматриваемой задаче, то кратность спектра оператора $T_{1}$ конечна при любом $\beta \in(0,1)$. Отметим, что если непрерывность функции $S$ нарушается хотя бы в одной точке, то может случиться, что $m\left(T_{1}\right)=\infty$ при всех $\beta>0$. Пусть $\mathscr{M}=\operatorname{dim} \operatorname{ker} S(0)$. Поскольку точка 0 является единственной спектральной особенностью оператора $T$ при $c \in \mathscr{E}$, $m\left(T_{1}\right)=\mathscr{M}$ при достаточно малом $\beta$, и это число - минимальное возможное. В то же время эффективная оценка угла между соответствующими инвариантными подпространствами не вытекает из абстрактных соображений. Как показывает следуюшая теорема, в которой собраны результаты изучения случая $c \in \mathscr{E}$, функцию $\gamma_{k}$ для нашей задачи можно выбрать таким образом, что $m\left(T_{1}\right)=\mathscr{M}$, а для спектральной компоненты $T_{\text {ess }}$, отвечающей окрестности спектральной особенности, угол оценивается в терминах оператора $Q$.

Пусть $\chi_{\delta}$ - оператор умножения на индикатор множества $\mathbb{R} \backslash[-\delta, \delta], \delta \neq 0$, в $L^{2}(\mathbb{R} ; \Delta)$. Тогда $\mathscr{P}_{\delta}=I-W \chi_{\delta} W^{-1}$ является спектральным проектором оператора $T$, отвечающим интервалу $[-\delta, \delta]$, в следующем смысле: (i) $\mathscr{P}_{\delta}$ - ограниченный оператор в $\mathscr{N}_{e}$ и $\mathscr{P}_{\delta}^{2}=\mathscr{P}_{\delta}$, (ii) $\mathscr{P}_{\delta}$ коммутирует с $R(z)$ при любом $z \in \rho(T)$ и, таким образом, $\operatorname{Ran} \mathscr{P}_{\delta}$ - инвариантное подпространство оператора $T$, (iii) $\sigma\left(\left.T\right|_{\operatorname{Ran} \mathscr{P}_{\delta}}\right)=[-\delta, \delta]$ и (iv) любое инвариантное подпространство $\mathscr{G} \subset \mathscr{N}_{e}$ оператора $T$, такое, что $\sigma(T \mid \mathscr{G}) \subset[-\delta, \delta]$, содержится в $\operatorname{Ran} \mathscr{P}_{\delta}$. Свойства (i)-(iii) являются следствиями леммы 1 и соотношения (1). Свойство (iv) вытекает, например, из наличия скалярного кратного [2, теорема VII.6.2].

ТЕОРема 2. Если $c \in \mathscr{E}$, то для любого достаточно малого $\delta \neq 0$ оператор $T_{\mathrm{ess}}$ может быть представлен в виде линейной суммы $T_{\mathrm{ess}}=T_{1}+T_{2}$ операторов $T_{1}, T_{2}$, действующих в инвариантных подпространствах $\mathscr{H}_{1}, \mathscr{H}_{2}$, таких, umo

1) $T_{1}$ имеет спектр $\sigma\left(T_{1}\right)=[-\delta, \delta]$ кратности $\mathscr{M}=\operatorname{dim} \operatorname{ker} S(0)$,

2) $T_{2}$ подобен самосопряженному, и $\sigma\left(T_{2}\right)=\mathbb{R}$,

3) $\left(\mathscr{H}_{1}, \mathscr{H}_{2}\right)>0$. Угол $\left(\mathscr{H}_{1}, \mathscr{P}_{\delta} \mathscr{H}_{2}\right)$ допускает оченку

$$
\sin \left(\mathscr{H}_{1}, \mathscr{P}_{\delta} \mathscr{H}_{2}\right) \geqslant p / \sqrt{2}, \quad p=\operatorname{dist}(0, \sigma(S(0)) \backslash\{0\}) .
$$


ДокАЗАтЕльство. Выберем $\delta \neq 0$ так, чтобы ранг спектрального проектора оператора $D(k)$, отвечающего промежутку $\left[0, p^{2} / 2\right)$, был равен $\mathscr{M}$ при всех $k \in[-\delta, \delta]$. Определим подпространства $X_{1}(k), X_{2}(k) \subset E$ в соответствии с конструкцией $\S 1$ с $\gamma_{k}=\frac{1}{2} p^{2} \chi_{[-\delta, \delta]}(k)$. По лемме $1 \mathscr{H}_{i}=\overline{W X_{i}}, i=$ 1,2 , - инвариантные подпространства оператора $T, m\left(T_{1}\right)=\mathscr{M}$, а сужение $T_{2}=\left.T\right|_{\mathscr{H}_{2}}$ подобно самосопряженному оператору. Действительно, $\left.S\right|_{X_{2}}$ имеет ограниченный обратный, так как $\sup _{k \in[-\delta, \delta]}\left\|\left(\left.S(k)\right|_{X_{2}(k)}\right)^{-1}\right\| \leqslant \sqrt{2} / p<\infty$ и $\sup _{|k| \geqslant \delta}\left\|S^{-1}(k)\right\|<\infty$ (см. доказательство предложения 3$)$.

Пользуясь оценкой (10) и формулами (14), (15), нетрудно оценить $\delta$ снизу. В результате получим, что

ЗАмЕчАниЕ. Утверждения пп. 1)-3) теоремы 2 справедливы при

$$
\delta<\frac{1}{2 a} \exp \left(-\frac{64 \Upsilon^{2}}{p^{2}} \frac{a c_{2}^{2}}{c_{1}}-\gamma\right)
$$

где $c_{2}=\|c\|_{L^{2}(\mathbb{R})}$ и $\Upsilon=\left(\int_{-1}^{1} \int_{-1}^{1} \ln ^{4}|x-y| d x d y\right)^{1 / 4}$.

\section{Заключительные замечания}

(а) В предельном случае изученной модели, когда $c(x) \equiv c_{\infty}$ при всех $x \in \mathbb{R}$, спектр оператора $T$ легко вычислить, используя преобразование Фурье по $x$. В результате получим $\sigma(T)=\mathbb{R} \cup\left[i c_{\infty}, 0\right)$. Этот факт выглядит естественно в том смысле, что для произвольного $c \in L_{0}^{+}$, полагая $c_{\varepsilon}(x)=c(\varepsilon x)$, будем иметь $N\left(c_{\varepsilon}\right) \rightarrow \infty$ при $\varepsilon \rightarrow 0$.

Заметим, что в рассматриваемом случае $T$ не является спектральным оператором (см. [14]) при всех $c_{\infty}>0$. Именно, можно показать, что спектральный проектор $\mathscr{P}_{\alpha}$ оператора $T$, соответствующий интервалу $\left[i \alpha, i c_{\infty}\right]$, ограничен при $\alpha>0$, однако $\left\|\mathscr{P}_{\alpha}\right\| \rightarrow \infty$ при $\alpha \rightarrow 0$, т.е. $T$ не обладает свойством $(\mathrm{B})$ спектральных операторов.

(b) Для диссипативности $L$ необходимо и достаточно выполнение условия подкритичности $c_{\infty} \leqslant 1$. В то же время оператор $T$ диссипативен при любом неотрицательном $c \in L^{\infty}$. Таким образом, в случае $c_{\infty}>1$ можно использовать (7) в качестве грубой оценки числа экспоненциально растущих мод для надкритичной задачи.

\section{ЛИТЕРАТУРА}

1. Шихов С. Б. Вопросы математической теории реакторов. Линейный анализ. Атомиздат, М., 1973.

2. Секефальви-Надь Б., Фояш Ч. Гармонический анализ операторов в гильбертовом пространстве. Мир, М., 1970.

3. Павлов Б. С. Об условиях отделимости спектральных компонент диссипативного оператора. Изв. АН СССР., сер. матем., 39, вып. 1, 123-148 (1975).

4. Набоко C. Н. Функциональная модель теории возмущений и ее приложения к теории рассеяния. Труды МИАН, 147, 86-114 (1980).

5. Lehner J. The spectrum of the neutron transport operator for the infinite slab. J. Math. Mech., 11, No. 2, 173-181 (1962).

6. Наймарк M. А. Исследование спектра и разложение по собственным функциям несамосопряженного дифференциального оператора второго порядка на полуоси. Труды MMO, 3, 181-270 (1954). 
7. Лянце В. Э. О дифференциальном операторе со спектральными особенностями. I. Матем. сб., 64 (106), вып. 4, 521-561 (1964); II. Матем. сб. 65 (107), вып. 1, 47-103 (1964).

8. Сахнович Л. А. Диссипативные операторы с абсолютно непрерывным спектром. Труды MMO, 19, 211-270 (1968).

9. Lehner J., Wing G. On the spectrum of an unsymmetric operator arising in the transport theory of neutrons. Comm. Pure Appl. Math., 8, 217-234 (1955).

10. Lehner J., Wing G. Solution of the linearized Boltzmann equation for the slab geometry. Duke Math. J., 23, 125-142 (1956).

11. Бродский M. C. Треугольные и жордановы представления линейных операторов. Наука, М., 1969.

12. Рид М. и Саймон Б. Методы современной математической физики, т. 4. Мир, M., 1982.

13. Павлов Б. С. Самосопряженная дилатация диссипативного оператора Шрёдингера и разложение по его собственным функциям. Матем. сб., 102, вып. 4, 511-536 (1977).

14. Данфорд Н. и Швари, Дж. Линейные операторы. Часть III. Мир, М., 1974.

Санкт-Петербургский государственный университет, НИИ физики, лаборатория теории сложных систем

Санкт-Петербургский государственный университет, физический факультет, кафедра математической физики Санкт-Петербургский государственный университет, НИИ физики, лаборатория теории сложных систем
Поступило в редакцию 2 февраля 1998 г. 